\title{
la información
}

\section{para la industria de la construceión, en España}

\author{
A. COMYN, jefe de la División de Información del I.E.T.c.c.
}

\section{simopersis}

En este trabajo se pretende subrayar la actual importancia de una eficiente información y documentación para el mejor funcionamiento de las empresas vinculadas con la construcción y sus materiales, especialmente con el cemento.

Se analizan datos estadísticos de la industria en España, y asimismo se recogen cifras referentes a una encuesta sobre empresas de la construcción en Inglaterra. Se da un énfasis especial a la labor del I.E.T., en cuanto a sus servicios de información, documentación y publicaciones.

\section{Introduceión}

A pesar del título, este artículo desea tratar con detalle únicamente de la labor de información y documentación del Instituto Eduardo Torroja de la Construcción y del Cemento. Ello quiere decir, que no consideraremos ahora la importante labor que desarrollan, además, los ministerios y organismos dependientes de los mismos, las escuelas técnicas, colegios profesionales y otras entidades públicas o privadas. Tampoco nos referiremos al Servicio de asistencia técnica del I.E.T., que, mediante sus estudios y ensayos, proporciona eficaz ayuda técnica a la industria.

\section{El problema de la comanicación}

Uno de los mayores problemas con los que cualquier organismo de investigación ha de enfrentarse, es el de asegurar la máxima difusión y aplicación posible a los resultados de sus trabajos. Esto es cierto incluso en los departamentos de investigación en empresas comerciales ordinarias, en donde podría pensarse que los problemas de comunicación tenderían a una solución más fácil.

El problema es muy acusado y principalmente debido a la estructura minifundista de la industria de la construcción en nuestro país (la cual no difiere esencialmente de la de otros muchos países), así como a su naturaleza ampliamente tradicional -aunque como en todas partes, esto vaya gradualmente decreciendo-. Como datos estadísticos diremos que en España existen unas 15.000 empresas constructoras, y de ellas solamente 37 tienen invertido en maquinaria más de 50 millones de pesetas. Ello explica que el nivel técnico del equipo de estas empresas varíe tan ampliamente como el número de empleados; las firmas pequeñas cuentan casi exclusivamente con personas preparadas algo más que a nivel de operarios especializados; las mayores empresas emplean a grupos de personas con titulaciones superiores. Hay en España cerca de 4.000 arquitectos, unos 3.000 ingenieros de caminos, y un total aproximado de otros ingenieros, licenciados y restantes titulados, relacionados con la construcción, del orden de 20.000, para los que, en mayor o menor medida, gran parte del trabajo del Instituto es de gran importancia. Señalaremos también que España dispone de más de 200 oficinas técnicas que agrupan a unas 2.500 personas, de ellas $40 \%$ titulados. Hay asimismo varios miles de fabricantes de materiales, y también desde la gran empresa a la muy pequeña y desde los relacionados únicamente con los materiales de construcción hasta los que tienen a la industria de la construcción como una más entre las que sirven. 
De un modo resumido diremos: que la población activa empleada en 1969 en la construcción supera las 1.200 .000 personas, esperando llegue a 1.500 .000 en 1971 .

Aparte de las personas mencionadas que se encuentran en la industria o profesiones, todas las autoridades regionales y locales en España son asimismo responsables de la construcción. Las empresas comerciales y de desarrollo financiero, también han de preocuparse de la construcción y conservación de edificios y obras de ingeniería civil. En el escalón más elemental están hasta el hombre o la mujer, habitantes de núcleos urbanos o rurales y que ocupan o trabajan en los edificios. Todo esto representa un público numerosísimo receptor potencial de la información sobre la construcción y, en un último análisis, de la información más aplicable o práctica sobre los resultados de la investigación.

Otras cifras que merecen consideración se refieren a las inversiones gubernamentales en construcción: En 1968 se dedicaron más de 51.000 millones de pesetas; en 1969 fueron 62.000 millones de pesetas, y para el año actual - 1970 - se ha previsto una inversión por el Ministerio de Obras Públicas de más de 67.000 millones de pesetas, de ellos 15.000 millones para obras hidráulicas y 13.000 millones para carreteras. Por su parte, el Ministerio de la Vivienda tiene programada la edificación de 350.000 viviendas.

\section{Aletividades de doenmentación e información del I.E.T.}

Es preciso decir, desde ahora, que, con respecto al I.E.T., muy poca de su actividad, en cuanto a información y documentación, está dirigida al público en general. Ocasionalmente se ha hecho, y con el resultado deseado. Pero es preciso darse cuenta de que las posibilidades del I.E.T., a este respecto, son limitadas, y al existir otros organismos bien capacitados ha de procurar defenderse ante una posible tempestad de preguntas que a cualquier nivel pudiera presentarse. A pesar de ello, 500 de entre 2.000 consultas escritas y un número aproximado de consultas telefónicas proceden de individuos no pertenecientes al aspecto técnico-científico de la construcción.

El I.E.T. utiliza, en la medida de sus disponibilidades, los canales usuales mediante los cuales la información puede llegar a contratistas, fabricantes y profesionales. Existen probablemente más revistas técnicas, dirigidas a los varios componentes de la industria de la construcción, que en otras industrias, y parte de la información del I.E.T. se publica también mediante estas revistas del ramo. En nuestra sede de Costillares se celebran frecuentemente conferencias para diferentes tipos de especialidades y dirigidas a distintos niveles de preparación, tanto por parte de investigadores del Centro como de técnicos de otros organismos o empresas comerciales. El equipo de investigadores del I.E.T. se desplaza con cierta frecuencia para dictar conferencias en provincias o en el extranjero.

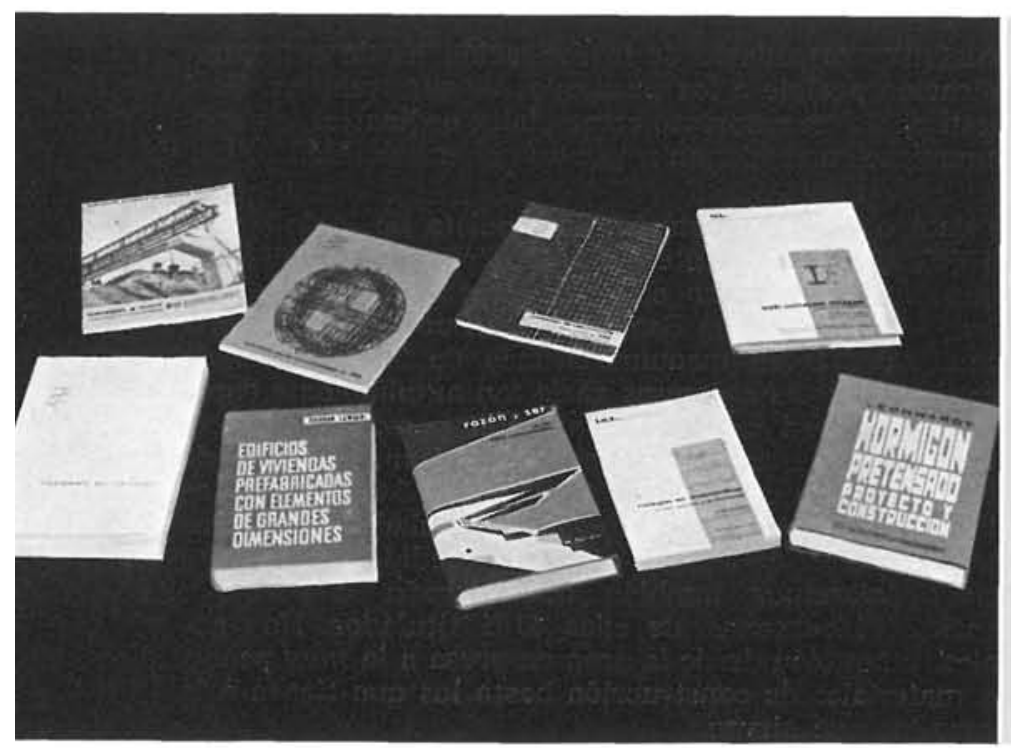

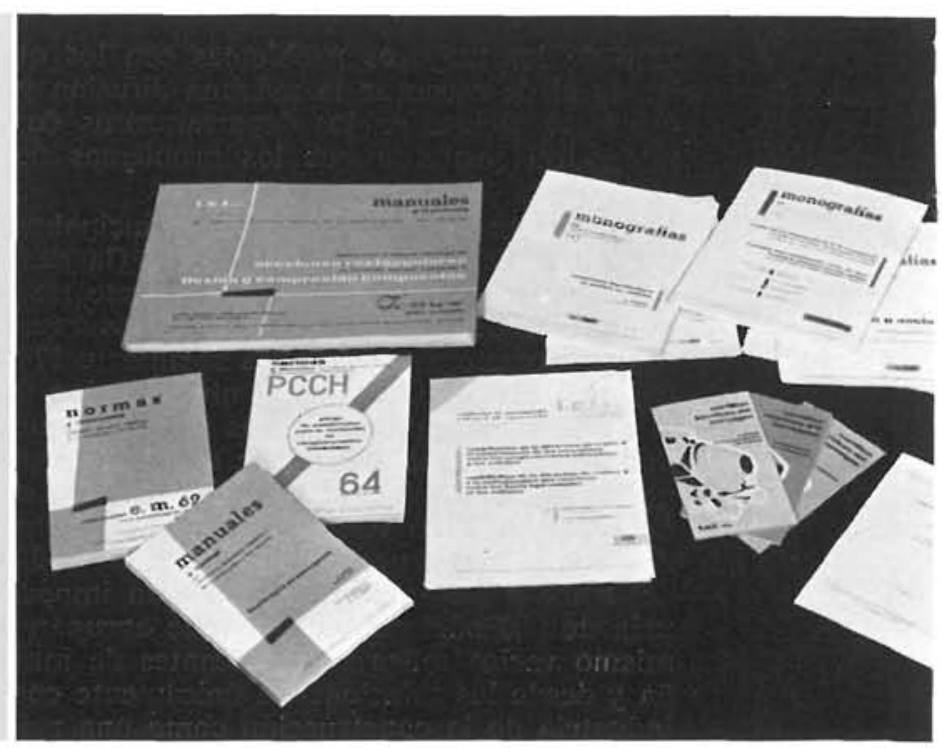

Otras publicaciones: manuales, monografías, cuadernos de investigación, cartillas técnicas del hormigón, etc. 
Gran parte de la labor del I.E.T. encuentra expresión en las diversas Normas e Instrucciones de carácter oficial. Además, una serie de publicaciones tienen la categoría de Códigos de Buena Práctica sin fuerza legal, pero reconocidos como útiles elementos de trabajo.

La más importante labor de difusión la realiza el I.E.T. a través de sus propias publicaciones, dirigidas con mayor o menor exactitud a las diferentes clases y niveles de usuarios. Desde los "Cuadernos de Investigación", serie bilingüie (español-francés o español-inglés), proyectados para expertos e institutos de investigación, hasta las "Cartillas de Hormigón», destinadas a encargados y obreros especializados, pasando por las revistas «Informes de la Construcción» (arquitectos, ingenieros y empresas constructoras), «Materiales de Construcción» (especialmente directores y técnicos de fábricas de materiales de construcción y particularmente de cemento) y las diversas series de Monografías, Manuales y Folletos. Mención aparte merecen los libros, partiendo de «Razón y Ser de los Tipos Estructurales», debido a la pluma de nuestro Fundador y primer Director, Prof. Dr. Ing. Eduardo Torroja, hasta llegar a los libros de texto para alumnos de E.T.S. y Cursos de Especialización.

Con carácter gratuito se envía trimestralmente el Boletín de Información, que llega ahora a 55.000 personas o entidades hablispanas, interesadas en su lectura.

El I.E.T., en general, la Sala de Lectura y la Biblioteca, en particular, son objeto de continuadas visitas por parte de diversas clases de usuarios, que acuden en busca de información de las más variadas especialidades y que reciben información en el momento o hacen la petición de una búsqueda más detenida.

Sobre este particular queremos subrayar que el I.E.T. dispone de medios para proporcionar informaciones bibliográficas e industriales sobre temas específicos relacionados con la construcción y sus materiales, con énfasis especial sobre el cemento. La solicitud puede hacerse personalmente o por escrito, y el servicio incluye asimismo la posibilidad de recibir xerocopias, así como traducciones de los trabajos que el usuario considere de interés.

Estas son las diversas facetas de la documentación e información que produce y transmite el I.E.T. No hay duda de que gran parte de ella llega al público para la que está destinada. También, sin duda alguna, cierta parte de la documentación no cubre los objetivos, debido a su especial naturaleza. El punto álgido del problema es no tanto maximizar la cantidad de información a emitir, como sí asegurar la mayor asimilación de sus resultados en la práctica diaria en la industria o por parte de las profesiones a que se destina.

Ahora bien, no es menos cierto que el interés por estar informados es aún pequeño. No se llega a valorar adecuadamente lo que ello representa para cualquier tipo de industria y es nuestro propósito que en la construcción se adquiera esta conciencia apreciando la eficacia de un trabajo muy útil, que supone mucho esfuerzo y dinero.

\section{Fuentes de información empleadas por las Empresas Construetoras}

Después de analizar resumidamente la amplitud de las actividades de información y documentación del I.E.T., consideramos ilustrativo y oportuno indicar el resultado de una encuesta, realizada hace ya más de seis años en Inglaterra, sobre cómo llega la información a profesionales y empresas de la construcción. Tenemos noticias recientes de que actualmente en Inglaterra, y justamente en los últimos cinco años, la información para la construcción ha encontrado su camino, brindado con su interés por profesionales e industriales a los documentalistas y servicios de información. Quizás las cifras pudieran variar de alguna manera al tratarse de una encuesta realizada en nuestro país, pero entendemos que las consecuencias a deducir no serán ciertamente extrañas.

La encuesta se basó en la consulta a 595 empresas constructoras inglesas que empleaban entre 20 y 500 operarios. Se concertaron 522 entrevistas con los directores o gerentes de las firmas, con un porcentaje de respuestas del $88 \%$. Esta elevada cifra testifica el interés común por el tema.

Aparte de las preguntas de tipo general sobre la importancia de la empresa y su tipo de trabajo, el cuestionario cubría las diversas fuentes de información empleadas por los que respondían, su conocimiento y uso de innovaciones específicas y sus procesos para resolver problemas y tomar decisiones. Hubo, como es natural, muy diferentes respuestas entre firmas. Las empresas mayores mostraban más interés por resolver los problemas y sobre los medios de solucionarlos, así como en lo referente a las nuevas ideas y su mejor uso. 

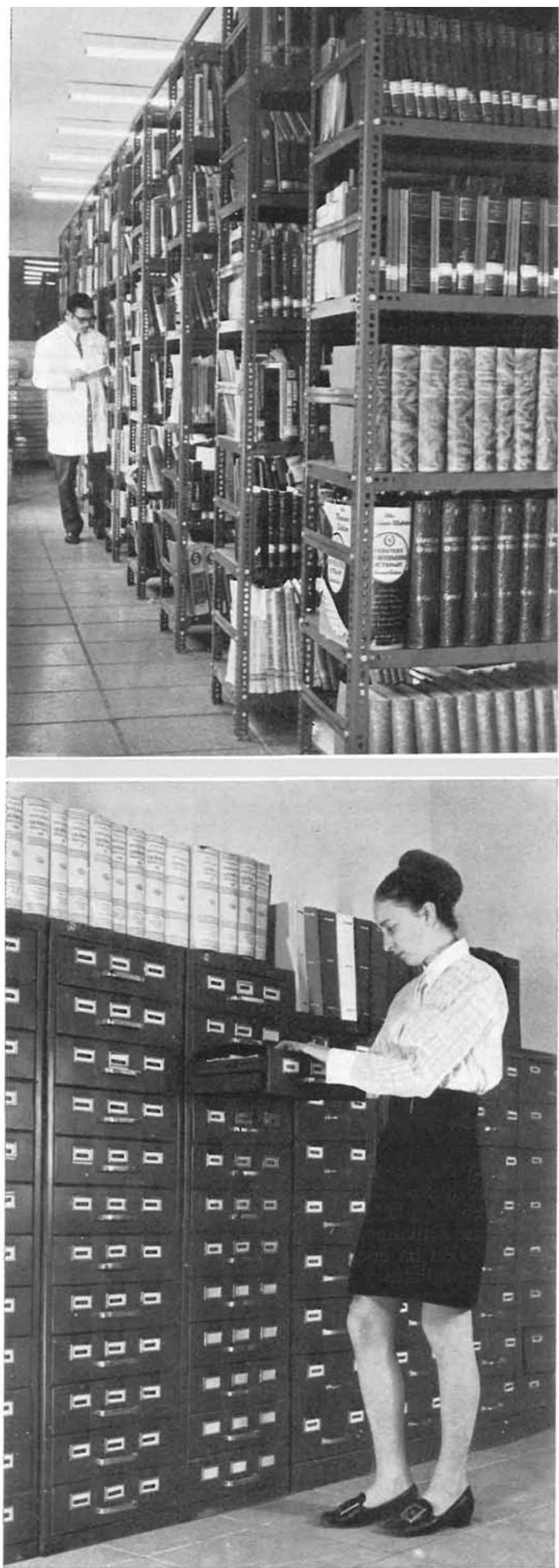

La biblioteca alberga más de 12.000 libros y 487 colecciones de revistas técnicas.

Los diversos ficheros contienen más de 1.000 .000 de referencias de trabajos, aparecidos en todo el mundo.

Las fuentes de información se consideraron teniendo en cuenta 15 epígrafes distintos, tales como revistas técnicas, exposiciones, conferencias, literatura comercial, consulta a suministradores, organizaciones de investigación, fuentes informales, etc. La variedad fue grande: 5 personas dijeron que no utilizaban ninguna fuente de información, mientras que 2 hacían uso de las 15 señaladas y cerca de los $2 / 3$ empleaban de 5 a 9 .

Las revistas técnicas con/o servicios de resúmenes, recibieron la máxima atención. El $40 \%$ les otorgó ser la primera fuente de información, mientras que la literatura comercial obtenía el $25 \%$. Las exposiciones, películas y conferencias conjuntamente, no llegaban al $8 \%$. Un $15 \%$ sólo leía una revista técnica. Los anuncios contenidos en las revistas recibieron también atención, ya que el $50 \%$ dijeron miraban todos los anuncios; mientras que el $40 \%$ se interesaba por las noticias, y un $25 \%$ leían todos los artículos técnicos. Casi un $25 \%$ de las firmas sacaban fichas de los temas de interés de las revistas, las cuales se conservaban como fondo de documentación.

Quedó patente que gran número de respuestas coincidía al señalar que acudían frecuentemente a los suministradores de materiales para resolver los problemas técnicos. Cerca del $75 \%$ había tenido contactos de este tipo. En un $50 \%$ de las firmas visitadas, un técnico superior de la firma había pedido información a la Building Research Station (organismo inglés análogo al I.E.T.). La proporción era mayor en las grandes empresas. Considerando solamente a directores o gerentes, el $34 \%$ había recurrido a la B.R.S. en firmas de hasta 50 empleados; el $55 \%$, en firmas de 50 a 100 empleados, y cerca del $70 \%$, en empresas mayores. Se intentó valorar la utilidad de las referencias recibidas, aunque sólo se logró saber que las relativas a pinturas y humedades resultaban menos satisfactorias, mientras que la información sobre hormigones y solados fue de la mayor utilidad. 
El más importante canal de comunicación de la B.R.S. son sus publicaciones y en especial los "Digest» (semejantes a las Cartillas Técnicas del I.E.T., pero quizás para niveles superiores). De 10 de ellos seleccionados, hubo dos relativos a juntas y condensaciones que resultaron imprescindibles para el $90 \%$ de las firmas, mientras que uno sobre grúas para edificación sólo interesó al $30 \%$.

Con respecto a las instrucciones oficiales, normas y documentos análogos, el $50 \%$ señalaron que los utilizaban como información, aparte de su empleo prescriptivo. Otras obras de referencia (manuales, libros científicos, etc...) fueron usados por $1 / 3$ de los consultados; unos 100 los usaban poco y un $40 \%$ indicó no contaban con tales libros.

Se intentó conocer la importancia que concedían a los medios no usuales de comunicación, como son el intercambio de experiencias o la tendencia a seguir un "modelo de empresa». Quizás un enfoque particular o la competencia mal estimada, impidió tener la oportunidad de conocer estos extremos.

En general, los nuevos adelantos técnicos y científicos eran conocidos y empleados con mayor frecuencia por parte de empresas que empleaban varias fuentes de información, pretendiendo de esta forma estar al día y creando, si era preciso, los servicios adecuados.

La técnica general revelada por esta encuesta es que la industria de la construcción es una industria pendiente básicamente de los problemas del momento, relativos a la realización y contratación, sin una clara preocupación por el futuro. Los problemas técnicos se reconocen solamente al producirse algún fallo u obstrucción del trabajo; y cuando se trata de un material, lo más frecuente es acudir al suministrador. En las empresas mayores, la información técnica de la B.R.S. siempre se ha tenido en cuenta. Es curioso notar que a través de la encuesta nadie reconoció la existencia de problemas de organización.

Como resumen de las respuestas reunidas, parece muy importante el que se deba tomar en consideración la mejora de la información para las industrias, adecuándola a la competencia e interés de las mismas. Por ejemplo, el material destinado a los contratistas ha de formarse con mensajes sencillos y directos para que realmente penetre. Más aún, el hecho de que los constructores no reconozcan la existencia de algunos problemas, no es fácil resolverlo por los medios convencionales de comunicación de masas; es preciso considerar más a fondo sus casos.

\section{Conclusiones}

Y hasta aquí los datos suministrados por la encuesta realizada en Inglaterra hace años, que entendemos pueden ser útiles datos a considerar con respecto a la industria de la construcción en nuestro país y que pueden resultar muy constructivos - valga la redundancia- al pensar en tomar medidas en cuanto a una mejor información técnica.

Es indudable que, ante la "explosión» de las informaciones, hay una serie de soluciones que se pueden arbitrar, y que, aunque no pueda precisarse adecuadamente el valor de la proporción COSTE/ EFICACIA de la información, sí puede hacer que ésta sea rentable para la marcha de la empresa. 


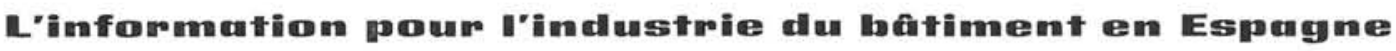

A. Comyn, chef de la Division d'Information de l'I.E.T.c.c.

Dans cet article, l'auteur veut souligner l'importance d'une information et documentation efficientes pour le meilleur fonctionnement des entreprises liées à la construction et à ses matériaux, en particulier à l'industrie du ciment.

L'auteur analyse des statistiques sur l'industrie en Espagne et donne des chiffres concernant une enquête faite sur des entreprises britanniques du bâtiment. Une attention spéciale est attachée à la tâche menée par l'I.E.T.c.c. en ce qui concerne ses services d'information, documentation et publications.

\section{Informention fon the comstrmetrion imalustrut in Spain}

A. Comyn, Chief of the Information Division of the I.E.T.c.c.

This paper seeks to emphasize the current importance of having adequate information and documentation so that the firms devoted to construction and construction materials, particularly cement, can operate more efficiently.

An analysis is provided of the statistical information of the construction industry in Spain, and figures are also given on fact finding enquiries undertaken in Britain in connection with construction businesses. Special reference is made to the work done at the I.E.T.c.c. in the department of information, documentation and publications.

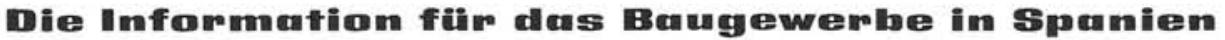

A. Comyn, Leiter der Informationsabteilung des I.E.T.c.c.

Diese Arbeit will die Bedeutung hervorheben, die heute einer wirksamen Information und Dokumentation zukommt im Hinblick auf ein besseres Funktioneren derjenigen Unternehmen, die mit dem Baugewerke und den entsprechenden Materialien, insbesondere dem Zement, in Zusammenhang steben.

Es werden statistische Angaben über die spanische Industrie analysiert und auch Auswertungen einer Umfrage unter Baufirmen in England gegeben. Besonders hervorgehoben wird die Tätigkeit des I.E.T.c.c. in Bezug auf Information, Dokumentation und Veröffentlichungen. 